\title{
Postoperative Regional Analgesia Options Following Esophageal Surgery
}

\author{
Pete L. Pelletier, MD, and Margaret E. Griesemer, DO \\ Department of Anesthesiology, Rush University Medical Center, Chicago, IL
}

Thoracic epidural analgesia (TEA) has long been considered routine care for postoperative pain management following open esophagectomy. ${ }^{1}$ Using an epidural catheter, a local anesthetic solution, with or without opioids or adjuvants, is infused into the epidural space. The principal site of action of local anesthetics in the epidural space is believed to be the spinal roots and cord; although, with prolonged infusions, redistribution of opioids likely contributes a supraspinal effect. Epidurals can provide excellent pain relief but they are associated with a failure rate of between 13 and $32 \%$ as well as adverse effects, including hypotension, urinary retention, and impaired mobility. ${ }^{2,3}$ Studies have suggested epidurals may increase the length of stay following thoracic surgery. ${ }^{4}$ In addition, due to the risk of epidural hematoma, therapeutic anticoagulation is not recommended while an epidural catheter is in place.

Along with thoracic epidurals, paravertebral nerve blocks (PVBs) have been shown, in prior studies, to provide effective analgesia following esophagectomy. PVBs provide ipsilateral somatic and symptomatic coverage over multiple dermatomal levels by depositing local anesthetic just lateral to the epidural space, between the parietal pleural and superior costotransverse ligament. Studies have reported on the efficacy of paravertebral nerve catheters in the thoracic surgery literature, particularly when combined with an opioid analgesic for breakthrough pain. ${ }^{5}$ It is common for the anesthesiologist to place paravertebral

(C) Society of Surgical Oncology 2021

First Received: 28 April 2021

Accepted: 10 May 2021;

Published Online: 26 May 2021

P. L. Pelletier, MD

e-mail: Pete_L_Pelletier@rush.edu catheters under ultrasound guidance in the preoperative area, thereby allowing for optimal perioperative analgesia. Prior thoracic surgery studies reveal a lower failure rate of PVBs and rare complications, including unwanted epidural spread of local anesthetic, hypotension, and pneumothorax. ${ }^{6,7}$

In this issue of Annals of Surgical Oncology, Feenstra et al. prospectively report on 25 patients undergoing thoracolaparoscopic Ivor Lewis esophagectomy compared with 25 retrospective patients who had undergone the same procedure. $^{8}$ In the prospective group, patients received paravertebral anesthesia (PVA) into the paravertebral space at the level of T4-T5 ipsilateral to the mini-thoracotomy. Following a $25 \mathrm{mg}$ bupivacaine bolus, an infusion of bupivacaine $0.125 \%$ was administered for 3 days. The retrospective group received TEA via a catheter placed between $\mathrm{T} 5$ and $\mathrm{T} 8$, and had a continuous infusion of $0.125 \%$ bupivacaine with $5 \mu \mathrm{g} / \mathrm{mL}$ sufentanil. The primary outcome of the study was highest numeric pain rating scale (NRS) pain score in the first $72 \mathrm{~h}$, while secondary outcomes included vasopressor and fluid requirements as well as length of hospital stay. The findings of the study demonstrated superiority of the TEA compared with the PVA analgesia, with a mean difference of 0.75 ; however, as noted by the authors, pain was reported as $\leq 4$ in both groups. Further examination of the findings suggests that pain control in the TEA group was superior to the PVA group for the first $24 \mathrm{~h}$ postoperatively, but there was no difference between the two groups on days 2 and 3. All the secondary outcomes demonstrated no difference between groups.

One notable difference between groups was that the TEA group received a combination of a local anesthetic and opioid solution, while the PVA group received a local anesthetic-only infusion supplemented with an opioid PCA. Despite this difference, the study demonstrated that 
PVA analgesia is effective and comparable with TEA for minimally invasive esophagectomy. This is a clinically important finding as the risks and complications of PVA are potentially less than TEA, while both offer acceptable pain control and PVA may decrease the length of stay compared with TEA. Additionally, this study suggests that administration of local anesthetic in a single paravertebral site is comparable with TEA, thereby refuting the belief that PVBs would only cover one dermatome above and below the injection site, thus necessitating multiple injections to provide wide dermatomal coverage.

Unfortunately, limitations of this study prevent the wide generalizability of the results. The study used a prospective/retrospective design around a change in clinical practice and did not include the effect of change in practice in their statistical analysis of the results. In addition, the sample size was small (25 patients per group) and the patients were not matched for clinical or procedural factors that could have impacted the outcomes. Inspection of the subject characteristics in the groups suggest that the TEA group was younger and had a lower body mass index (BMI) and American Society of Anesthesiologists (ASA) classification, while the PVA group had less radiation therapy but more previous abdominal surgeries. Ideally, with only 25 patients per group, matching could have been performed to minimize the confounding impact of these variables and increase the certainty of the differences between groups based solely on the difference in analgesia delivery.

Nevertheless, in our opinion, the largest limitation of the study is the differences in opioid strategies between the two groups. With the TEA group having sufentanil in the epidural infusion and the PVA group having an opioid PCA, it is difficult to directly compare the pain control between these groups. Sufentanil is an opioid with both high lipid solubility and mu-receptor affinity, which promotes rapid onset of intense analgesia when administered in the epidural space. This difference in opioid administration between groups resulted in the TEA group receiving more opioids, with a mean difference of $68.7 \mathrm{mg}$ morphine equivalents. The use of a second analgesic in the continuous infusion of the TEA group significantly limits the reader's ability to determine if this difference alone could account for the observed difference of improved analgesia during the first $24 \mathrm{~h}$ postoperatively.

Finally, any discussion of regional anesthesia for the chest wall would be incomplete without mentioning the erector spinae plane block (ESPB), which is performed by depositing local anesthesia just outside the paravertebral space, between the erector spinae muscles and the transverse process. The ESPB is thought to be a safer technique than the epidural or paravertebral block due to the local anesthetic injection being further away from the spinal canal and parietal pleura. There is a paucity of data on the utility of ESPB in esophagectomy, however it is demonstrated to be effective for post-thoracotomy pain following lung resection. ${ }^{9,10}$

Adequate postoperative pain control in thoracic surgery is imperative to decreasing complications and minimizing the development of a chronic postoperative pain syndrome. Conversion from acute to chronic postoperative pain following thoracic surgery is approximately $40 \%$ in open thoracotomy and $30 \%$ in thoracoscopy. ${ }^{11}$ The best way to minimize chronic postoperative pain is accomplished through improvements in surgical techniques and adequate multimodal pain control, as demonstrated by the development of enhanced recovery after surgery (ERAS) protocols. As minimally invasive esophagectomy becomes more prevalent, additional studies are needed to determine the optimal modalities for pain control and ERAS. This study could represent a paradigm shift from the traditional TEA for esophagectomy to peripheral nerve blocks such as PVBs or ESPBs. We anxiously await additional studies examining the efficacy of various regional anesthetics in the setting of minimally invasive esophagectomy, as well as in the wider range of thoracic and abdominal surgical procedures.

\section{REFERENCES}

1. Carli F, Kehlet H, Baldini G, et al. Evidence basis for regional anesthesia in multidisciplinary fast-track surgical care pathways. Reg Anesth Pain Med. 2011;36:63-72.

2. Manion SC, Berennan TJ. Thoracic epidural analgesia and acute pain management. Anesthesiology. 2011;115:181-8.

3. Gleicher Y, Singer O, Choi S, et al. Thoracic epidural catheter placement in a preoperative block area improves operating room efficiency and decreases epidural failure rate. Reg Anesth Pain Med. 2017;42:649-51.

4. Dunham W, Lombard F, Edwards D, et al. Effect of regional analgesia techniques on opioid consumption and length of stay after thoracic surgery. Semin Cardiothorac Vasc Anesth. 2020. h ttps://doi.org/10.1177/1089253220949434.

5. Zhang W, Fang C, Li J, et al. Intravenous sufentanil analgesia in patients with esophageal cancer undergoing combed thoracoscopic-laparoscopic esophagectomy: a safe and effective alternative. J Cardiothorac Vasc Anesth. 2014;28:966-72.

6. Yeung JH, Gates S, Naidu BV, et al. Paravertebral block versus thoracic epidural for patients undergoing thoracotomy. Cochrane Database Syst Rev. 2016;2:CD009121.

7. Naja MZ, Lonnqvist PA. Somatic paravertebral nerve blockade: incidence of failed block and complications. Anaesthesia. 2001;56:1184-8.

8. Feenstra ML, Hoope TW, Hermanides J, et al. Optimal perioperative pain management in esophageal surgery: an evaluation of paravertebral analgesia. Ann Surg Oncol. 2021. https://doi.org/10. 1245/s10434-021-10172-1 
9. Pirsaharkhiz N, Comolli K, Fujiwara W, et al. Utility of erector spinae plane block in thoracic surgery. J Cardiothorac Surg. 2020;15:91

10. Huang W, Wang W, Xie W, et al. Erector Spinae plane block for postoperative analgesia in breast and thoracic surgery: a systematic review and meta-analysis. $J$ Clin Anesth. 2020;66:109900.

11. Arends S, Böhmer AB, Poels $M$, Schieren M, Koryllos A, Wappler F, Wappler F, Joppich R. Post-thoracotomy pain syndrome: seldom severe, often neuropathic, treated unspecific, and insufficient. PAIN Rep. 2020;5(2):e810. https://doi.org/10.1 097/PR9.0000000000000810.

Publisher's Note Springer Nature remains neutral with regard to jurisdictional claims in published maps and institutional affiliations. 